\title{
Die Konfession des Herzogs Albrecht von Preussen vom 13. Juli 1554.
}

\author{
Veröffentlicht von Frang Koch.
}

Während des Osiandrischen Lehrstreites hatte Herzog Albrecht von Preaßen die Wurttembergischen Theologen anfgefordert, ein Gutachten za senden, weil er hoffte, dadurch die streitenden Parteien versöhnen za können; aber seine Erwartungen erfullten sich nicht.

Als anch die sächsische Gesandtschaft') im Jahre 1553 keine Einigang erzielte, wandte er sich an den Herzog Christoph von Wurttemberg mit der Bitte, ihm einige Theologen zu senden, um durch mundliche Verhandlangen die Ursache des Streits za beseitigen. Am 12. Mai 1554 trafen in Königsberg ein: D. Jacobus Beurlin and D. Rapertas Durr. Zwei Monate darauf - am 13. Jali 1554 erschien das vorliegende Bekenntnis Albrechts, durch das er sich von dem Verdachte, nicht mehr Anhänger der Angsburgischen Konfession za sein, reinigen wollte.

Der Vorlage des nachfolgenden Abdrackes bildet eine von zwei Händen hergestellte Abschrift in dem Folianten S. 59 Nr. 8 p. 953 bis 986 der Königsberger Stadtbibliothek. Sie stammt ans dem Nachlasse Joachim Mörlins and warde ihm nach Brannschweig zar Kenntnisnahme gesandt. Von dort hat er das Aktenstlick, als er 1567 zun samlkindischen Bischof gewählt wurde, nach Königsberg gebracht, wodurch sich das Vorhandensein in der Königsberger Stadtbibliothek erklärt. Ohne Zweifel ist die Abschrift von Freunden Mörlins hergestellt.

1) Vgl. meine Arbeit: Die sächsigche Gesandtschaft zu Königgberg während des Osiandrischen Lehrstreits im Jahre 1553 in der Altpreußischen Mlonatsschrift Bd. 40 Heft 3 und 4. 
Salig, dem die Konfession aus einer Handscbrift der Wolfenbutteler Bibliothek bekannt war, hält Herzog Albrecht fur ihren Verfasser. „Er war, sagt dieser Reformationshistoriker, ein gelehrter Herr. studierte sehr fleiBig and scbaffte sich auch eine zahlreiche Bibliothek an, and also ist kein Zweifel, dab er sie selbst gemacht ${ }^{1}$ )."

Diese Auffassung ist rein änBerlich und anzutreffend. Herzog Albrecht kann nicht der Verfasser sein, weil er keine so umfassenden theologischen Kenntnisse hatte, um solch ein ausführliches und eingehend begründetes Bekenntnis aufzustellen. Es unterliegt keinem Zweifel, daB die Konfession aus dem Kreise der Konigsberger Hoftheologen hervorging ${ }^{2}$ ), die sich des Namens ihres Landesherrn bedienten, um dem Werke größeren Nachdruck zu verleihen ${ }^{3}$ ).

1) Vollstäudige Historie der augsburgischen Konfession (Halle 1733) S. 1027. „Ich hätte fügt Salig hinzn, „die Konfession gern ganz mit eindrucken lassen, wenn sie nicht zu weitläufftig wäre. Denn sie ist vortrefflich und zeigt des Herzogs wahres und ungeheucheltes Christentum." Jedoch gibt er a. a. 0. S. 1027 bis 1029 eingehend den Inhalt an.

2) So auch Hartknoch, PreuB. Kirchenhistorie S. $365 \mathrm{f}$.

3) Anmerknng der Redaktion. DaB die erwähnte Wolfenbütteler Handschrift (7. 9. Aug. 2) nicht herangezogen wurde, ist nicht die Schuld des Verfassers, dem vielmehr ein auf Uebersendnng der Handschrift nach Wolfenbüttel gerichtetes Gesuch einfach abgeschlagen wurde. Aber auch dem Herausgeber, der sich dann an die genannte Bibliothek mit deln Ersuchen wandte, die Handschrift zu seiner Benutzung an das ihm unterstellte Stettiner Staatsarchiv zu senden, wo er fïr sachgcmäße Aufbewahrang etc. Sorge tragen werde, erging es nicht viel besser. Er wurde durch folgendes Reskript beglückt:

„Euer Hochwohlgeboren. Die Verleihung von Handschriften ist nur mit spezieller Genehmigung Herzoglichen Stratsministeriums gestattet and nur an öffentliche, unter direkter stantlicher Aufsicht stehende Bibliotheken; in dem Gesuch ist eine kurze Darlegung des Zwecks der beabsichtigten Benutzung za geben und eine Erklärang des Vorstandes der entleihenden Bibliothek, da $B$ er die hiesigen Verleihbedingungen anerkennt, beizüfügen. Ob das Herzogliche Staatsministerium in diesem Falle eine Ausnahme machen und gestatten wird, die Handschrift 7. 9. Ang. 2. an das dortige Stastsarchiv zu verleihen, da in Stettin eine öffentliche Staatsbibliothek nicht ist, wird von der Begründung abhängen, die Sie dafür vortragen werden. Mit größter Hochachtung (gez.) Milchsack. Oberbibliothekar." 
Von Gottes guaden unser Albrechtendes eltern II arggrafen zu Brandenburgetc. in PreuBen etc. Herzogen Konfession.

Gottes gnad und fried darch Jesum Christum unsern einigen heiland wunschen wir allen fromen gutherzigen Christen und zuforderst unsern lieben underthanen.

Wie der heilige Apostel Petrus alle Christen vermanet, daB sie alle Zeit bereit sein sollten zar verantwortung einem Jeden, der grund fordert des glaubens oder der hoffnung, so in jnen ist, auch der Herr Christas wil und gebeat, daB wir jnen fur den Menschen bekennen sollen, Item daB wir wahen sollen, dieweil wir nicht wissen die stunde, darin der Herr komen und uns aus diesem leben abfordern wirt: haben wir uns höchstes fleisses bemuhet von der Zeit an, da wir anfenglich vormittelst gotlicher gnaden zum erkentnis der warheit des heiligen Evangelii gekomen seindt, anf daB wir in unser lehr und bekentnis also gegrundet und gefasset weren, damit wir derselben halben nicht allein fur Gott ein gut gewissen hatten, sondern auch unsern widersacher dester weniger za furchten hatten, uns auch nichts weniger vorsehen, denn dab uns jemand eines abfals von derselben einmahl erkanten und bekanten warheit solle beschuldiget haben, welchs doch aber gleichwol von etlichen wider unser vermuten und verschulden mit unserm grossen beschwer und vieler gatherzigen Christen nicht geringen ergernis geschehen; denn nachdem sich Kurz vorschinen Zeit zwischen unsere Theologen etliche harte dispatationes und zwispalt uber dem Artikel der rechtfertigung zugetragen, haben inen dieselben unsere vorleumder und widerwertige darvon arsach genomen uns als einen verirreten and abtrunnigen von der rechtschaffenen Christlichen lehre fast im ganzen reich Teadscher Nation auszutragen und meniglich hohes und nidriges standes einzubilden, Dartuber auch die Kirchen unsers furstenthams jemerlich and gantz beschwerlich zerruttet and

Dab ich es abgelehnt babe, dew letzten Satze des Schreibens gemäß Herrn Oberbibliothekar Milchsack zum Richter darüber zu machen, ob mein Gesuch Berücksichtigung verdiene oder nicht, versteht sich wohl von selbst. Andererseits habe ich geglaubt, das Schreiben hier mitteilen zu sollen als ein Beispiel der bedauerlichen Rückständigkeit, in der sich im Punkte der Handschriftenrersendung die Wolfenbütteler Bibliothek immer noch gefällt. Vielleicht hilft diese „Flucht in die Oeffentlichkeit" dazu, daß endlich Wandel geschafft wird. 
verunruiget, die underthanen zu vielen widerwillen wider einander rerhetzet und endlich auch zu mercklichem ungehorsam gegen uns gereizet und bewogen worden.

Dardurch wir denn hochlich verursacht und gedrangen nicht allein fur unsere Person und unsers gewissens halben mit fleis nachzaforschen, wie es am die streitigen Artickel. daruber dieser unfried und spaltung erwachsen, sich verhielte, sondern auch ein offentlich bekentnis zu thun am darvon auszugeben, daraus meniglich rerstehen moge, welches lehre wir bisher anhengig gewesen, bey welcher anch wir durch Gottes gnad verharret und bis an unser end zu verharren bedacht und entschlossen, Den almechtigen, ewigen gatigen Gott ron herzen bittend, er wolle uns durch seinen heiligen Geist In solcher seiner warheit alzeit gnediglich regiren, leiten und bewaren Amen.

Auch thun wir von allen menschen, wes standes die Immer sein nogen. den dieses anser bekentnis vorkamet, freundlich und gnediglich begeren, Nachdem sie die unstrefliche gotliche lehre, der wir uns hiermit In warheit zugethan und anhengig bekennen, hirin befanden werden, wollen uns doch ferners verdachts und auflage nicht allein fur Ire Person erlassen, sondern auch bey andern befreien, auf da $\beta$ dem beschwerlichen ergernas, so sich ans solcher bezichtigang and vieler leat ungegrunden und unbillichen lestern und afterreden rerursacht, endlich einmal geweret und die Kirchen widerum zu Christlichem fried and vereinigung darch gottes gnedige hulf und verleihang mogen gebracht werden, dazu Gott am seines heiligen namens ehre willen um vieler betrubten gewissen trostes and heils willen seine gnade nach seiner grossen barmherzigkeit reichlich geben wolle. Amen!

Und nachdem in gegenwertiger Zwispalt, so sich uber der lehre ron der rechtfertignng des Sunders erhoben, alle disputation entweder die Person unsers herrn and heilands Jesu Christi oder sein ampt als seine wolthaten gegen uns, sein thun, leiden und sterben and also auch das werck der erlösung and rechtfertigang belanget, wollen wir erstlich uns erkleren, was wir vermoge der heiligen schrift von der Person unsers herrn Jesu Christi halten and glauben, Nachmals auch von den andern stucken unser christlich bekentnis darthun.

Und bekennen hirmit far Gott and Jeder maniglich, daB wir vestiglich glauben, dab Jesus Christus unser her sey, der ewige eingeborne Son Gottes von ewigkeit von Gott dem vater geboren, warer Gott von warem Gott, gleicher gewalt, Kraft, herlichkeit und wesens mit Gott dem vater und heiligem Geist, wie denn solchs anch im Symbolo Nicenc, gefasset and in der schrieft Klerlich gegrundet: 
Psalm 2: Du bist mein lieber Sohn, heut hab ich dich gezenget.

Joann. 3: Also hat Gott die welt geliebet, daß er seynen eingebornen Sohn gab.

Joann. 8: Ehe dan Abraham war, bin ich.

Michä 5: Aus dir (Bethlehem) sol mir Komen, der In Israel her sey, welchs außgang von anfang und ewigkeit her gewest ist. etc.

Joann. I Im Aufang war das wort und das wort war bey Gott and das wort war Gott.

Dieser ewige und eingeborne Sohn Gottes ist mensch worden, empfangen vom heiligen Geist, geboren aus Maria der Jungfrauen, wie Lucas am 1. and 2. beschreibet and Joan. am 1. Das Wort ist fleisch worden etc. Und ist dieser Jesus Christus warer Gott und mensch in einer Person ein einiger Christus und heiland aller, die auf ihn tranen, and ist auch nach seiner menschlichen natur gerecht and rein, das ist on alle Sunde und dem gotlichen gesetz und willen Jn allen seinen Kreften gleichformig and gehorsam; nach seiner gotlichen natur aber Ist er die ewige wesentliche gerechtigkeit selbst, wie er auch ewige wesentliche macht, leben, weisheit, liebe and gutigkeit ist, daron spricht Zachar. 9: Dein König Komt za Dir ein gerechter and helfer.

Paul. 2. Cor. 5: Den Christam, der von Keiner sünde wuste.

Lnc. 1: Das heilige, das von dir geboren wirt ete. Jerem. 23: Man wirt ihn nennen her unser gerechtigkeit etc.

Und damit wir ans dieses Pancts von vereinigung beider naturen In Christo noch weiter erkleren (um etlicher ungeschickter rede und gedanken willen, welcher sich bey werender Zwispalt etliche haben vernehmen lassen) Ist dieses weiter anser meinung and bekentnis:

Wen wir sprechen, Gott ist mensch worden, Ist es nicht zu verstehen, dab die Gotheit oder gotliche natur in die menschheit sey vorwandelt worden; denn also wer er nicht warer Got blieben. Desgleichen, wenn wir sprechen, Jesus ist der ewige ware Sohn Gottes, sol man nicht gedenken, daB die menschbeit oder menschliche natur in die Gotheit verwandelt sey. Denn also were er auch nimer ein mensch, sondern also ist es zu verstehen, dab diese beide Naturen die Gotliche und die menschliche in Christo also vereiniget seind, daß sie nicht zwo personen, sondern beide eine einige person seind und in ewigkeit nicht Konner von einander geschieden noch getrennet werden, und bleibt doch Gott Gott and mensch mensch; aber um dieser allerhochsten vereinigung willen wirt von dieser Person Jesu Christi das alles recht und mit warheit gesagt, daß da sonst von der gotlichen 
oder menschlichen natur Christi In sonderheit mag gesagt werden. Als dieser Jesus (hristus ist schopfer himels and erden. Ist der ewige Sohn Gottes, Ist warhaftiger ewiger Gott. Item Gott hat für uns gelitten und sein bluet fur uns vergossen, wie denn der Apostel Sanct Paulus redet Act. 20: Der heilige Geist hat euch gesetzet zn Bischofe"I zu weiden die gemeine Gottes, welche er durch sein eigen bluet erworben hat. Also sagt man recht nach der schrieft: Got ist von der Jungfranen Maria geboren. wie auch Lue. 1 geschrieben ist: Du Maria wirst einen Sohn geberen, der sol ein Son des aller hechsten heissen. Von solcher vereinigung beider Naturen und solcher weib zu reden, welche man communicationem idiomatam nennet, baben die alten anter anderen auch dieses, wie wol schwache iedoch nicht unbequeme gleichnis geben: wie in einen gluenden eißen Zwo naturen Zusamen komen nemlich das feuer und das eißen, welche auch also voreiniget werden, daB, deweyl es gluet, eisen und feuer ein Kuch ist und wirt, doch das eisen nicht feuer and das feuer nicht eisen, Also sind and pleiben in Christo zwo Unterschiedene naturen die Gottheit und die Menscheit In einer person rereinigt, von welcher person dieser naturen keine in ewigkeit wirt abgesondert.

Es ist aber diese gleichnis daram za gering und zu schwag, deweil die Zusamenfugang des eisens und feuers nicht eine person macht, auch leichtiglich widerum mag von einander gescheiden werden.

Also gebrauchen sie dis gleichnis noch weiter: wie das eisen vom feuer erleuchtet, erhitzet und zubereitet wirt, dab es sich las arbeitten wie man will, Also wirt die Menschliche Natur in Christo von der Gottlichen durchleuchtet, daB die Gottheit leuchtet in der ganzen Menschlichen natur Christi, thut die wunder, werk und allen wolgefelligen willen seines himmlischen Vaters.

Und wie man von dem gluenden feurigen eisen alles das, was man sonst von ihrer beider wesen zu reden pfleget. sagen mach als: das ist eisen, das ist 8chweer, das ist feuer. das leuchtet, das brennt ete. Also sagt man recht ron der person Christi alles das, so der Gottlichen oder Menschlichen Natureigenschaft ist als: Dieser ist ein wahrer Mensch. dieser ist der gecrenzigte Gott, dieser ist Gottes ewiger sobn, dieser hat himel and Erden geschaffen.

Dises ist nu unser glaub und bekantuns von der person unsers herren Jesu Christi, und ist solches alles in heiliger schrift reichlich gegrundet; denn es auch Gottes ernster wille ist, daß wyr den Sohn Gottes also erkennen und ehren sollen und durch ihn den vater, auch in solcher erkentnis 
ron tag zu tag zunehmen und berzlichen trost und freud daran haben, daß sich die gottliche Maiestet also genediglich mit unser schwachen natur derselben widerum aufznhelfen voreiniget hat, wollen nuhn von seinem Ampt and wolthaten gegen uns unsern glauben erkleren und anzeigen.

Diser Sohn Gottes ist Mensch worden (wie gesagt) und in die welt komen, die sunder sehlig zu machen wie $S$. Paulus spricht 1. Timoth 1. Denn das ist je gewislich wahr und ein theares werdes wort, daß Christas Jesus komen ist in die welt, die sunder selig za machen und Christus Joan. 12 cap. Ich bin nicht komen, da $B$ ich die welt richte, sondern daB ich die welt selig mache, denn also hat Gott die welt gelibet, $\mathrm{da} B$ er seinen eingebornen Sohn gab. auf dab alle, die an ihn glauben, nicht verloren werden. sondern das ewige leben haben Joann 3. Die ursach aber. darum die welt eines solchen mittlers und heilands, der zugleich Gott und Mensch wehre, bedarfte, Ist furnemlich diese. daß wyr durch die sund und Ubertretung in thod und Gottes Zorn gefallen wahren und vermochte kein Creatur uns aus solcher not nemlich von Gottes Zorn und ewigem thod za erlosen, und daiegen das leben, die gerechtigkeit, herlichkeit und ewige sehligkeit, der wyr verlustig worden wahren, wideram zu erwerben, viel weniger vermochten wyr selbst uns der sunde, so in unser Natur stecket, aus eigen Kreften los zu machen, Gott volkomenen gehorsam zu leisten and also Gottes Zorn zu stillen und der ewigen straf zu entgelien.

Darum bat Gott selber, der da Reich ist von barmherzigkeit und an des sunders thode keinen gefallen hat, sich unser angenohmen und seinen eivigen Sohn zu einem mittler verordnet und uns geschenkt, $\mathrm{daB}$ er durch sein allerheiligstes, thearstes opfer und Blatvergißen Gottes gerechten Zorn versonete, auch sein ewiges unwandelbares gesetze, deweil es ihn ron Menschen solte gehalten und erfullet werden, volkomlich fur uns erfallete, um uber dis auch die straf des thodes, die wyr verdinet hetten, erlitte und doch im thod nicht bliebe, sondern denselben durch gottliche Kraft uberwunde und rom thod auferstund und allen, so in ihn glauben, widerum das leben und ewige sehligkeit schenkte. Solche stuck alle samt haben ihre klare Zeugnis in heiliger schrift, dab wyr alle sunder sind und kinder des Zorns von Natur Psal. 5: Sehet, ich bin aus suudlichem samen gezeuget and meine Matter hat micb in sunde empfangen.

Röm. 3. Sie sind alzamal sunder and sie mangelen der berlicheit Gottes.

Archiv far Reformationggesehichte V. 2. 
Von der Erlosung aber und ron dem gehorsam oder der Erfullung des Gesetzes. durch ('hristum für uns geschehen. reden diese volgende spruche:

Math. 5 . Ieh bin nicht komen das Gesetz aufzuheben. sondern zu erfullen.

Philipper 2. Jesus Christus, ob er wol in Gottlicher gestalt wahr, hielt ers doch nicht fur einen raub Gott gleicb sein, sondern eußert sich selbst und nam knechtes gestalt an, ward gleich wie ein andrer Mensch und an geberden ein Mensch erfunden, erniedriget sich selbs und war gehorsam bis zum thod ja zum thod am Kreuz. Gal. 4. Da aber die Zeit erfallet wahr, sandte Gott seinen sohn, geboren von einem weibe und unter das gesetz gethan, auf daB er die, so unter dem gesetz wahren, erlosete, daß wyr die Kuntschaft empfingen Joann. 17. Ich heilige (das ist ich opfere) mich fur sie etc. Heb. 10. Mit einem opfer hat Christus in ewigkeit volendet, die geheiligt werden.

Iath. 20 des Menschen Sohn ist nicht komen, daß er ihm dienen lasse, sondern dab er diene und geb sein leben zar Erlosung fur viele 1. Joann. 2: Jesus Christus ist die versohnung fur unser sunde und nicht allein fur die unser, sondern auch far der ganzen welt.

Und in der einsetzang des $\Lambda$ bentmals des herren, dis ist mein leib, der fur euch gegeben wirt, dis ist mein blut, das fur euch und fur viele vergossen wirt zu vorgebung der sunden. Röm. 8. Gott hat seines einigen sohns nicht verschonet, sondern hat ihn fur uns alle dahin gegeben. heilet.

Jsa. 53. Petr. 3: Durch seine wanden sind wyr ge-

Gal. 3 Christas hat uns erlöset rom Fluch des gesetzes, da er ward ein fluch fur uns.

1. Joann. 1. Das blut Jesu Christi macht uns rein von allen sunden.

Joann. 1. sihe, das ist Gottes lam, welchs der welt sunde tregt.

Von der herlichkeit aber, die uns Christns widerbracht hat, zeugt Christus selbst:

Joan. 17. Ich hab ihnen gegeben die herlicheit, die da myr gegeben hast.

Aus disen und dergleichen spruchen ist nuhn leicht $z u$ ersehen, wie hoch and teuer dieser gehorsam and das leyden und sterben des herrn Jesa Christi zu achten, deweyl die schrift klerlich lehret and zenget, da $B$ uns dardurch vergebung der sunden und die Erlosung von Gottes Zorn and strengem gericht widerfahren and erworben sei.

Jedoch ist daßelb anch leichtlich daher Za erweisen, dieweil die person, die da leidet and stirbet, nicht allein Menseb, 
sondern auch warhaftiger ewiger Gott ist. Daraus denn je folgen mus. daB solch leiden und sterben einer solchen hohen Gottlichen person hoher mus geachtet sein. denn als sonst die ganze welt litte; denn so viel Gott grober ist denn die ganze welt, so viel ist auch Christi leyden und Thod wirdiger, denn sonst aller naturen sein mochte, deweil Christus, der fur uns leidet und stirbet, zugleich auch wahrer Gott selbst ist. Solchs aber wollen wyr nicht also vorstanden haben, als ob Christus nach seiner Gottlichen Natur gestorben sei, denn also wehr der Thod mechtig uber das gottliche wesen worden, welchs unmuglich ist, sonderen also glauben und bekennen wyr, daß Christus wahrer Gott and Meusch gestorben sei nach seiner menschlichen Natur warhaftiglich und sei begraben. Die Gottliche Natur aber ist nicht gestorben, Ist aber anch nicht von der Menschlichen Natur gesondert worden Im leiden und sterben, sondern in der personlichen vereinigang blieben und hat geruhet, wie $\therefore$. Jreneus redet, Also daB sie ihr Gottliche kraft nicht gebraucht hat, der Menscheit zu helfen, auf daß die Menscheit sterben kunte, welche sonst von wegen der voreinigung mit der gottlichen Natur, und daß sie ohne alle sund gewesen, nicht hatte sterben konnen, wo Christus nicht hatte williglichen die sterblichkeit an sich genohmen und fur uns sein leben zu lassen und ein opfer za werden beschlossen, wie er spricht Joan. 10: Niemaud nimt mein leben von mir, sondern Ich lasse es von mir selber, Ich hab es nicht zu lassen und wider zu nemen.

Item wie alle schmach, so Christo in seinem leiden begeznet, den ganzen Christum, der gott und mensch ist, betreffen, wirt solche leiden groß and Gotts leiden; Denn ob schon die -gottheit nicht stirbet, so leidet sie doch die schmach von den Juden und beiden und wird also mit der menscheit vorlestert und verspottet, und entlich das fleisch, welchs mit Gott in einer person vereyniget ist, ans Kreuz geheftet und getotet, daher denn die schrift spricht Act. 20: Gott habe die gemeyne durch sein blut erworben und S. Petrus 1. Petr. 4: Christus hab im fleisch fur uns gelitten.

Nach dem nhun Christus den 'Tod fur uns gelitten und das gericht auf sich selbst genomen hat, Ist er auch nider gestigen zur hellen und hat helle, Tod und Teufel durch seine almechtige Kraft uberwuuden and ist vom Tode wider auferstanden am dritten tage nach der schrieft Psalm 16: Da wirst meyne sel nicht in der hell laßen noch zu geben, daB dein heiliger verwest, Hat also alle unsere feinde Ihrer macht beraubt und ein Schauspiel aus lhnen macht Col. 2. daß wyr uns nymmermehr fur Jhrer macht furchten durfen, wenn wir an Christum glauben. 
Jenn nachdem diese hohe theure bezalung und erlosung fur das menschliche geschlecht durch Jesum Christum geschehen ist, erfordert auch das Evangelium von allen menschen den glauben und lehret Klerlich, daß solche erlosung niemand zu gut Komen denn allein denen. die an Christum glauben. Nemlich daß er auch Jhr messias oder beiland sey. durch welchen Jhnen vergebung der sunden und die erbschàt des ewigen lebens erworben und geschenkt sey.

Derhalben, ob gleich der gehorsam das leiden und sterben (hristi unaussprechlicher Kraft und wirdigkeit ist, (wie oben gesagt) also, daß dadurch eine genogsame und uberschwengliche bezalung fur aller welt sunden geschehen, so ist es dennoch gottes wille und ordenung, daß allein die. so dem Evangelio glauben, dardurch sollen von jhren sunden abgewaschen und ron gottes Zorn und dem ewigen tode erloset sein.

Lnd darum hat Gott das Predigtampt verordnet und eingesetzt, durch welchs er das Evangelium aller welt verkundigen lest und gebeut allen menschen dasselb zu horen und mit glauben anzunemen, wie die stimme des ewigen vaters rom hiemel selbst lantet: Dis ist mein lieber Sohn. an dem ich wolgefallen habe, den solt ir horen.

Ind Christus Marc. 1: Thut busse und glaubet dem Evangelio.

S. Johannes der Teufer Joann. 3: Wer an den Sohn Gottes gleubet, der hat das ewige leben. Wer dem sohn nicht gleubet, der wirt das leben nicht sehen, sondern der Zorn Gottes bleibet über ihm.

Et ibidem Christus: Also bat gott die welt geliebet. dab er seinen Eingebornen Sohn gab, auf dab alle, die an ihn glauben, nicht verloren werden, sondern das ewige leben haben.

Item: Wer an mich gleubet, der wirt nicht gerichtet: wer aber nicht gleubet, der ist schon gerichtet.

Mar. 16: Wer glaubet und getauft wird, der wirt selig werden; wer aber nicht glaubet, der wirt verdamt werden.

Röm. 1: Das Evangelion ist eine Kraft Gottes, die da selig machet alle, die daran glauben.

Wir glauben und bekennen auch, daB solch predigtamt dareh den heiligen geist erhalten und Kreftig gemacht wirt, welcher durch das mundliche wort und durch den gebrauch der heiligen Sacrament Darzu auch durch seine gotliche Kraft und Innerliche wirkang die herzen der menschen ruhret, bewegt und erleuchtet, daß sie demselben glauben und sich warhaftig zu got bekeren und selig werden.

Und dieweil wir denn solchem allem, so uns Gott in seinem warbaftigen wort und heiligen Evangelio geoffen- 
baret und verheissen, von herzen durch Gottes gnad gleuben, auch in unser Kindheit jm nahmen des raters Sohns und heiligen Geistes getauft seind. so glauben wir auch und haben bey uns das Zeugnis des heiligen Geistes, daß wir endlich ein Kind Gottes und erbe des ewigen leben seind, Sintemal Gott in seinem Sohne wabrhaltig ist und nicht luegen Kan. der da spricht: Wer dem Evangelio gleubet und getauft wirt, der sol selig werden. Derhalben warten wir nuhn in gedult, darch die Hoffnung der seligen erlösung aus dem fleisch, welche gescheen und volkomentlich volendet werden wirt, wen wir auch nach dem fleisch in den tod Christi, darin wir getauft seind, der sunden absterben und am Jungsten tag wideram in herlichkeit aufersteben werden Zum ewigen leben, da wir denn mit Christo dem berre und allen seinen auserwelten leben and herlich sein werden in ewigkeit. Das ist gewislich war! Dem almechtigen ewigen gutigen Gott sey far solch seine unaussprecbliche gnade and barmherzigkeit lob und Dank gesagt in ewigkeit amen!

Also baben wir bisher in einer Karz and einfeltiglich erzelet and dargethan unser bekentnis ron dem Artikel der Rechtfertigung, Das ist, wie ein mensch von wegen Jesu Christi bey Gott zu gaaden komme, vergebung der sunden, den heiligen Geist und die erbschaft des ewigen lebens erlange, wie solchs S. Paulus zu den Römern furnemlich und zu den Galatern beschreibet und nach der lange ausfuret. Haben derhalben keinen zweifel, sondern seind ganz gewis ans gottes wort, daß es die warhaftige eintreshtige, der alremeinen Christlichen Kirchenlehre sey, achten auch, dab eben die, so in dieser Zwispalt wider uns gewesen, sich nicht widerstehen werden. darinnen etwas zu strafen oder zu ver(lanımen. Da es aber gleichwol hieruber von Jemand gescheen wurde, mussen wir desselben urteil als unrecht und Gotlos nicht achten und bej der erkanten und seligmachenden warheit nichts destoweniger bleiben.

Dieweil uns aber Gott mehr der ursach zu gnaden annimt, die sunde vergibt, den heiligen geist und erbschaft des ewigen lebens schenket durch Jesum Christum, daß wir furthin in unser angebornen bosheit bleiben. leben und verharren sollen. sondern vielmehr darum dab wir den alten menschen von tag zu tage abtöten und im geist unsers gemutes erneuert werden und anzihen den neuen Menschen, der nach Gott geschaffen ist in warer gerechtigkeit und heiligkeit, wie s. Paulus lehret Eph. 4., wollen wir birvon unsere meinung auch anzeigen.

Und erstlich diese unsere verneuerung von der versönung deutlich zu unterscheiden, mogen wir sie mit D. Luthero wol nehmen den andern theil der recbtfertigung in 
dem rerstand, wie $D$. luther dasselb gethan, im j1. Psalm. denn damit meinet und rerstehet er des heiligen geistes bad (wie er es auch nenuet). Das ist die reinigung und heiligung. dadurch wir von tag zu tage von sunden gereiniget und in gotseligem wandel zunehmen und gebessert werden, welche ans dem glauben in Christum, dadurch wir mit Gott versonet, als ein frucht herfur wachsen und folgen solle. Wie dan $S$. Paulus diese rerneuerung etwa gerechtigkeit gemeiniglich aber frucht der gerechtigkeit pflegt zu nehnen.

Nur so Jemand meynete, dab dieses auch geferlich ser: Nemlich diese verneuerung ein theil der rechtfertigung zu nehmen um mißverstandes willen, so sich etwan leicht daraus gebiren macht, Als ob nemlich die versonung durch Christum erworben nicht solte sein und bleiben die vollkomene rechtfertigung des sunders fur Gotts gericht, wenn er sich deraelben mit glauben annimt und trostet, sondern dazu mahl were der sunder ron Gots strengen gericht allererst volkomen gerechtfertiget, wen er auch befunde, wie er mit der that ernenert und ron sunden gereiniget were, wollen wir mit niemand uber solchen worten und wie dieses stuck aufs deutligst zu nennen sey, Zanken, wissen anch, daB es recht and zu heilsamer underichtang der bloden gewissen dinstlich sey, daß underschidlich und mit gater bescheidenheit ron beiden teilen geleret werde, wie wir denn derbalben auch selbst hirmit underschiedlich unser bekentnis davon thun und begeren auch hochlich, daß es diejenigen, so in predigtamt sind, hirin an Keinen fleis und bescheidenheit mangelu lassen, Damit nemlich diese beide stuck von der versonung und von Jren frncbten dem rolek on underla $\beta$ treulich furgetragen und wol eingebildet werden, und nicht da eins on das ander getriben, entweder der trost des Evangelii vertunckelt oder aber auf der andern seiten $\mathrm{Zu}$ fleischlicher sicherheit und unbusfertigem leben und also zu ewiger rerdamnis der menschen ursach gegeben werde.

Zum andern ir wol nahn wie vermeldet ans $S$. Paulo za Eph. am 4. cap. von den gleubigen die verneuerung erfordert wirt, so ist doch mit fleis za merken, daß solchs nicht darum geschihet, daB wir Gots strengen Zorn damit stillen, gnad fur ihm, vergebong der sunden und ewiges leben erwerben, denn dieses widerferet uns allen darch den glauben an Jesum Christum, sondern es seind weit andere und doch auch nicht geringere ursachen, daram Gott von den gleubigen solche verneuerung baben wil, Als nemlich sein unwandelbarer wille nnd ordnung, dab die sunde genzlich in anser natur aufhere und dagegen warer gehorsam gegen ihm und gerechtigkeit angerichtet werde, dieweil er nicht ein Gott ist, dem gotlos wesen gefalle psal. 5 . Item, auf dab durch 
unser sundlich und ergerlich leben Gottes wort nicht verlestert werde, sondern daß wie $\mathrm{S}$. Paulus zu Tito schreibet, wie die lehre unsers heilands Jesu Christi Zieret mit guten werken. Item damit wir nicht durch sunden wider gewissen vom glauben und ron der gnade widerum abfallen, den heiligen geist betruben und ausstoßen und also in Zeitliche und ewige strafe Gottes fallen, und was dergleichen mehr ursachen in der schrift, daß man gut werk thun solle angezogev, welche von unnoten thut weitleuftiger zu erzelen. Zum dritten, Ist nun hir weiter die frag, woher ein mensch das vermogen bekomme, Gott dem herren also in gerechtigkeit und heiligkeit uod in einem neuen wandel zu gehorsamen, Dieweil wir von natur solchs uicht vermogen. Darauf ja unser antwort und bekentnis, daß solchs von Gott sey, der den gleubigen zugleich mit rergebung der sunden auch den heiligen Geist gibt und also selbst wesentlich in uns wobnet anch Kreftlich wirket sie zu erleuchten und zu allem gehorsam, den Got in seinem wort erfordert, zu neigen und zu treiben.

Haben dieses unsers glaubens gewissen grand and Zeugnis in allen spruchen, die von sendung des heiligen geistes und von einwonung Gottes in menschen lebren als Joann. 14: Wer mich liebet, (spricht Christus) der wirt mein wort halten und mein vater wirt in lieben und wir werden zu ibm Komen und wonung bey Ihm machen.

1. Cor. 3: Wisset ihr nicht, daB ihr Gottes tempel seid, und daß der heilige geist in euch wohnet.

Wisset ibr nicht, da $B$ eaer leib ein tempel des heiligen (ieistes ist, welchen ihr habt von Gott?

Röm. 8: Ihr aber seid nicht fleischlich, sondern Geistlich, so anders Gottes geist in euch wonet; wer aber Christi geist nicht hat, der ist nicht seyn.

Gal. 4: Weil Ihr denn Kinder seit, hat Gott gesandt den Geist seynes Sohnes in euer herzen, der schreyet: Abba lieber vater.

Zum vierden, dieweil wir aber dureh die sund also verderbt sind, daß auch, nachdem wir durch Christum mit Gott versonet und durch den glauben Tempel Gottes worden sind, dennoch sunden in uns ubrig bleiben, so felt von der Gleubigen unvolkomenen werken und geborsam diese frag furr, Wie sie damit für Gottesgericht bestehen, Gott gefellig und angeneme seind?

Darauf antworten und bekennen wir: Es ist notig, daß ein mensch nach empfangener gnad ernstlichen willen und fürsatz habe, (welchen er dann gewiblich haben wirt, wo er durch erleuchtung des heiligen geistes seyne sund and gebrechen und den unermeblichen Zorn Gottes gegen der 
sund beide aus dem Gesetz Gottes und aus den. daß Keyne genugthuung für die sund bat gelten mogen, denn das unschaldige bitter leiden des Sohnes Gottes, rechtschaffen erkennet). Gott gehorsam $z a$ seyn und wissentlich wider seynen willen nicht zu handeln, und den glauben also in seynem leben und wandel zu uben und zu beweysen. Er sol auch erkennen und Jhm leid seyn lassen, daß noch so viel sund und gebrechen Jn seyner natur ubrig seind, damit die buebe nymermehr in diesem leben aufhore und fleischliche sicherheit nicht überhand neme. Endlich aber und zugleich sol dieser glaub ohne unterlaß im herzen leuchten und das gewissen trosten und anfrichten, daB, wie wir bey Gott anfenglich $\mathrm{Zu}$ gnaden Komen, also auch nbumebr in gnaden seyn und furhin allezeit darin bleiben, allein am des einigen mittlers Jesu Christi willen, der fur alle unsere stinde genugsame bezalung gethan, Gottes gerechten Zorn wider die sund dadurch Fersonet und gestillet hat, und vertrit uns noch ohn unterlaß sitzend zu der rechten seynes himlischen vaters, Also dab Jhm Gott auch unsern wiewol schwachen und unvollkomenen gehorsam gefallen lest um des treuen Christi willen, zu welchs rolkomenheit er unser unvolkomenheit im glauben ansihet und fur angeneme opfer rhumet. Wie S. Petrus sagt: Opfert geistliche opfer, die gott angenehme sind, durch Jesum Christum usw. Linter solchem gnaden schirm haben wir uns gar Keynes Zorns noch verdamnis fur gott, um unser schwacheit und unvolkomenheit willen. so wir noch in uns fulen, und gern loß seyn wolten, auch nach usserm vermogen derselben widerstehen und dempfen. zu besorgen, Denn so ein Cbrist nach erkanter und empfangener gnad so kleinmtitig sein wolte, dab er um seyner uberbleibenden und anklebenden sund willen, die er doch nicht in Jhm heischen lest, sondern durch den Geist Christi dawiderstrebet, und dieselb durch Gottes hulf ganz abzutilgen begeret, an der versonung mit gott Zweyfeln und retlich an seyner ewiger seligkeit verzweifeln wolte, der thete uber alle ander sunden, auch diese allerschrecklichste sunde, daß er gott in seyner gnedigen Zusage, allen busfertigen sundern getban, lugen straft, und dazu seyne unwirdigkeit und sunde achtet mechtiger sein dann den gehorsam, die wirdigkeit, heiligkeit und gerechtigkeit des Sobns gottes, dadurch er ans erloset und bey seynem himlischen vater diese gnade erworben hat, daß derselbige sein gehorsam und gerechtigkeit auch uns zugerechnet, und wir um derselben willen gerecht gesprochen und nicht zum tode verarteilt werden, sondern zur ewigen seligkeit erhoben.

Lnd um solches waren trostes willen ist es sehr gut und notig nbur wol und mit allem fleis zu betrachten die 
wirdigkeit unsers lieben mittlers und erlosers und seynes heiligen vordinstes, daß er nemlich selber warer got ist und also die wesentliche Einige gerechtigtigkeit, gegen welcher Jn Christo dem Ern aller Menschen ..... wiewol sie gros und schrecklich sind nichts zu achten, denn wer in diesem herlichen bochzeit Kleide und unter solchem treflichem gnaden schirm für Gott erscheinet, dem seind alle seyve sunde iberreichlich bedeckt und vergeben und wird ron gott dem vater ein solcher menseh nicht anders denn ein glidmaß seynes lieben Sohns, dem er also durch den glauben eingeleibet ist, geliebet. Also haben wir bißher anher angezeiget und unsern glauben bekant, Erstlich, von der person unsers allerliebsten Ern und heilandes Jesu Christi und Jn Jhm beider der Gottlichen und menschlichen natur unzerthreplicher voreinigang. Und zum andern von seynem hohen Amt und unaussprecblichen wolthaten, in dem er die glaubigen mit seynem Creuz and tod von sunden erloset, Gott mit Jhnen versonet, alle gnad und ewiges leben erworben hat, Und dab er sie, nach dem von gott zu gnaden angenomen, durch seynen geist vorneuert, und in diesem leben anfehet von der sunden unreinigkeit $Z a$ reinigen und gerechtigkeit in Jhnen wirklichen zu uben, welchs angefangen werk aller erst im Zukunftigen leben volkomlichen volendet und ewiglich bestehen und bleiben wird.

Aus diesen unser bekentnus, wiewol verstendige Christliche herzen, genugsamen bericht und Rechenschaft unsers waren glaubens werden empfangen sich auch selbst besoheidenlich wißen za berichten, $J n$ was verstand wir in dem .... von anser Rechtfertigung diese wort gebrauchen, Got oder das Gotliche wesen in Christo, oder Christus auch nach seyner gotlichen natur ist unser gerechtigkeit. Doch dieweil die spaltung von dem artickel der rechtfertigung sich iber dieser reden unsers verstandes far nemlich erbaben, und wir hieruber sehr unchristlich verlestert worden, Also daß man uns beschuldigt, wir trenneten beide naturen in Christo, wir vernichteten das teure blut und unschuldig leiden Jesu Christi und dergleichen andere gotslesterung uns $\mathrm{Zu}$ gewießen worden, und derbalben als verneinetenn oder verleugueten solchs wir, haben etliche wider uns gestritten, das leiden, sterben und gehorsam Christi sey unsere gerechtigkeit, Jtem die verzeihung der sunde sey unsere gerechtigkeit, demnach wollen wir zu mehrer unser entschuldigung unser vorgehenden gethann bekentnus uns dieser rede halben in sonderheit noch beßer erkleren. Und aufs Kurzte davon zu reden, wen wir sprechen: Got oder gotlich weßen oder gotliche gerechtigkeit in Christo ist unser gerechtigkeit, hat es nicht diese meinung, daß das gotliche weßen unser selber 
weBen. art oder natur sey oder mit unser menschlichen natar personlich vereinigt sey, oder da got außerhalb Christo oder abgesondert ron dem mensehen in Christo unsere gerechtigkeit sey, sondern dieses ist unsere meinung: Got in Cbristo ist unsere gerechtigkeit. das ist Gotts eingeborner Son. der Got selbst ist, und also fur sich selber und von Natur die wesentliche gerechtigkeit, dieser wirt aus gnaden auch unsere gerechtigkeit, nachdem er uns rom vater geschenket und $\mathrm{Zu}$ eigen gegeben worden, und wir um seynet willen $\mathrm{Zu}$ gnaden angenommen werden und dem vater wolgefallen. dieweil er in Christo mit der menschlichen natur in einiger person vereinigt und um derselbigen personlichen und unzertrenlichen vereinigung willen, mit und in der menschlichen natur alles gethan und gelitten hat nach dem willen Gottes seynes himelischen vaters, welchs zu thun and zu leiden zu unser erlosung von noten war, also ist er unser gerechtigkeit, und eben um der gotlichen natur willen in Christo, hat allen geborsam und leiden sterben und blutvergießen dieser person diese grobe Kraft and wirdigkeit. $\mathrm{da} B$ es eyne genugsame bezalung fur aller welt sunde ist, daß nur ein ieder, so den gekreuzigten Christum oder (damit wir es ia deutlich und ansdrucklich genug reden) den gecreuzigten Got in Christo durch den glauben erkent, annimt und mit sich fur gottes gericht bringt. der bestehet fur gottes gestrenger gerechtigkeit (die auber Christo den sundern ein verzerend feuer ist) unsersehret, sie wolte ihm sich selber verzeren, das unmuglich ist.

Von diesem der gleubigen allerhohesten trost und freidigkeit fur gottes strengen gericht, den sie in Got und der gerechtigkeit gottes in dem gecreuzigten Cbrist durch den glauben ergreiffen und faßen sollen, hat D. Martinus Luther oftmals in seynem schriften geleret, sonderlich aber, da er in der Postil uber das Evangelium Math. 16, Das man am tag Petri und Pauli lieset, also spricht: Wer auf dem felsen Christi gebanet ist. und in ergreift. der ist auf got gebanet, and ergreift der gerechtigkeit gottes, die Gott selbst ist, welche er nicht verwerfen Kan, denn sonst muß er sich selber verwerfen.

Welche nu also durch Got selbst in Christo Gotsgericht und Zorn ansgestanden und uberwunden haben und mit Got versonnet seint, in den selbigen wonet nu auch Christus durch den glauben, also daß er die glaubigen mit der that von sunden reinigt und sie freuen und heilig mache, wie oben von anderem teil der rechtfertigung gedacht worden.

Und dab Christus auch dieser gestalt oder im solchenn verstande der gleubigeu gerechtigkeit muge genennet werden, nemlich als der, der durch seyne gotliche Kraft and wirkung 
die verterbte menschliche natur widerum aufrichtet, mit seynem geist begabet. und die durch Adam wider verlorne gerechtigkeit widerbringt, das ist, mit der that von sunden reiniget, gerecht, from und heilig macht, solt ir billig bey allen Christen ungewweyfelt sein, dieweil die schrieft offentlich leret, daß Got durch Christum uns solche wolthat erzeige, nemlich selbst im menschen wone und kreftiglich wirke, und also durch seine Gotliche gerechtigkeit die natur verneuere, wie den auch oben Etliche Klar zeugnis der schrieft hierron erzelet werden. Und Cbristus spricht Joann 15: Wer in mir bleibt und ich in ym, der bringt viel frueht, den on mich Kont ir nichts thun. Und Paulas Philip 2: Got ist, der in euch wirket beide das wollen und das volbringen. Also redet auch $\mathrm{D}$. Martinus Luther sher deatlich daron in der Postil uber die Epistel am Christag Hebr. 1 uber diese wort: Lind hat gemacht die vereinigung unserer freaden durch sich selbst, spricht D. Luther: Wie durch sich selbst nemlich, daB er unser sunden auf sich genomen hat, am heiligen creuz wie Esaia 53. Aber dis ist anch noch nicht genug, sondern auch also durch sich selbst, daß wer da glaubt an in, dab er solchs fur uns gethan hat, durch und um deßelbigen glaubens willen, wonet er selbst in uns und reiniget uns teglich durch sein selbst eigen werck. Hacc Lutherus.

Ind in diesem verstande sagen wir anch, daß Got selbst unsere ware ewige gerechtigkeit sey, nachdem gewis ist, daß wie got in diesem leben von anfang machet die natur zu reinigen und zu verneuern, also wirt Got in der auferstehung der toten, die sunde warhaftiglich und volkomliglich in unser natur abgetilget sein und wirt der mensch in allen seinen Kreften, volkommener reinigkeit, heiligkeit und gerechtigkeit mit Gottis weisheit und willen haben, wie dan die schrieft sagt: Got wirt alles in allen sein. Jst also dieses alles der obgesetzten ler von der rechtfertignng nichts entkegen noch abbruchig, denn wir uns hiemit wollen deutlich erkleret haben, wirt dieses alles nicht von der gerechtigkeit, dardurch ein sunder $\mathrm{Zu}$ gnaden Kumt und fur gottis gericht bestehet (welchs eigentlich die gerechtigkeit des Evangelii ist) gemeinet, sondern von der verneuerung der natur, welche oben der ander teil der rechtfertigung ist genennet worden, und eigentlich nach dem gesetz gerechtigkeit ist, und genennet wirt, welche, dieweil sie Got in uns, in diesem leben anfebet und in dem Zukunftigen volendet, und ewiglich darzu uns erhelt, so ist auch selber Dieser ursach halben unser ware ewige gerechtigkeit.

Dieweil denn diese rede wie wir sie mit D. Luthero reden und verstehen: Got selbst in Christo ist unsre gerech- 
tigkeit, den armen betrubten gewißen uber alle mas trostlich ist, den handel der rechtfertigung in Keynem wege schedlich noch abbruchig und weder der h. scbrift noch Gotseligen lehrer gebrauch und gewonheit entgegen, haben wir sie auch nicht wiben zu verwerfen. darzu so gar die Naturen in Christo nicht getrennet noch seynes heiligen leiden und sterben geschendet wirt, daß wir eben damit die beiden naturen in Christo aufs aller sterkste zusamen faBen, sein leiden aber sterben und blutvergiben aufs aller heiligste herlichste, das des waren lebendigen Gottes leiden und blut mit S. Paulo rhumen und preißen und dasselbige fur Gottes strengen gericht trosten, und allen unsern feinden, sund, tod, bell and Tenfel damit trotzen und obsiegen.

Aus wolchem allen denn leichtlich abzunemen, weu wir sagen: Got in Christo ist unser gerechtigkeit, daß wir weder die menschliche Natur noch der selben gehorsam creuz, leiden und blatvergieBen darzu auch die vergebung der sunde, so er dardurch uns bey Gott erworben hat, nicht ausschließen, sondern alles wollen mit eingescbloben haben, und wenn gesagt wirt: die vergebung der sunden sey unsere gerechtigkeit fur Got, ist uns nichts entgegen, sondern glauben and bekennen, dab es recht und nach eigenschaft des Evangelii eigentlich und dentlich geredet sei. wißen auch. daß D. Martinus Luther oftmals der gleichen geredet und geschrieben, und ist doch dieses der obgesetzten rede: Got in Christo ist unsere gerechtigkeit, in solchem verstand ist nichts zu wider Denn alhie in diesen worten vergebung der sund ist unsere gerechtigkeit fur Got, wirt gerechtigkeit dasienige genennet. welchs uns der herr Christus durch seinen gehorsam, durch sein leiden und sterben ie verdienet und erworben. Und ist eben dieses. daß sich ein blodes grewiBen allezeit, so oft es mit got handeln sol, furnemlich rhumen und trosten mus, in diesen worten aber: Got in Christo ist unsere gerechtigkeit, wirt Klerlich die ursach angezeigt am welcher willen, des herren Christi leiden und sterben eyne genugsame teure bezalung uns diese gnade zu erwerben (daß nemlich Got denselben ganzen geborsam von Jesu Christo waren Got und menschen geleistet als von uns selber geschehen uns zurechnet und in ansehung desselben ans unsre sunde gnediglich vergibt and uns ... gefallen lest) hat sein ......, den wir oben zam oftern mal gemeldet, erhoet zu solchen hohen werk nemlich uns von sunden abzuwaschen. Gottis gerechten Zorn wider uns zu stillen and uns mit im zaversonen, nicht eynes Pur lautern menschen leiden und blutvergießen, sondern eyner solchen furtrefflichen und waren Gotlichen Person, daher den auch S. Paulus dasselbe dardurch wir erkauft seind, im blut Gottis nennet Act. 20. 
Nu wir mal die verzeihung der sunden durch den gehorsam Christi erworben. nach der lerhe des Erangelii unsere ware gerechtigkeit fur Got ist, hat es dennoch damit (wie oben auch Klerlich vermeldet) diese meinung auch nicht, dab es ein solcher gerechtfertigter und mit Got versonter mensch, so lang er in diesem leben ist, nach nichts anders trachten oder von Got entfangen solle, sondern es bei der vergebung der sunden an alle verenderung und verneuerung seyner natur und wandels bleiben lassen. den damit wir es ia dentlich geben, so ist es unleugbar, da $\beta$ nach der lerhe des gotlichen gesetzes gerechtigkeit ist, und beist die frommigkeit und tugend, welche ist ein volkomener gehorsam gegen allen geboten Gottis, zu welchen den gegehoret und notig ist volkommene reinichkeit der natur, und das die selbige an alle unreinigkeit der natur, and das dieselbige an allen gebrechen and boße zaneigung sey, wie aufenglich der mensch geschaffen und fur dem pfal geweben, diese gerechtigkeit und gleichformikeit mit dem willen gottis in seynem gesetz offenbart, wiederum in unser natur anzurichten, werden uns unsere sunde von Got von wegen Jesu Christi vergeben, dab wir also widerum eine wonung oder tempel Gottis werden, wie unsere ersten eltern für den fal gewesen sint, and wir also entlich auch mit der tat ron sunden genzlich gefreiet and dargegen die gerechtigkeit des gesetzes in uns angerichtet und geflanzet werde.

Diese verneuerang mus von anfang anch diesen .... haben (wiewol es leider schwechlich zagehet) and maßen die Kinder Gottis ire gliedmas nicht der ungerechtigkeit, sondern Got zu waffen der gerechtigkeit mit fleis darreichen und die sunde in irhen fleisch nicht hersehen laben, Nach diesem leben aber, da got alles in allem sein wird, wirt solche gerechtigkeit sampt der ganzen selikeit ire volkorneuheit haben und erreichen.

Solchs haben wir hiemit von unsers glaubens im artikel der rechtfertigung grand and gelegenheit meniglich wollen zu verstehen geben, genzlich verhoffend, es werden hieraus alle ware Christen rornemen und befinden, $\mathrm{da} B$ wir Keynen neuen unchristlichen glauben angenomen, sondern daß wir bey der waren christlichen (und wie mans ietzum nennet) der Augspurgischen Konfession verwanten Kirchenlehre ohn alles wancken geblieben, denn wir auch mit Gott zeugen mögen and wollen, dab wir weder Osiandro noch andere in anderen verstand beyfall gegeben haben, sie nhun in Jhren reden wiederschrieften andere meynung von der rechtfertigung gehabt und unter die Christen ansbreiten wollen, ist es unsere meynung garnicht, sie hiermit zu entschuldigen oder 
zu verthedigen, sondern laßen sie selbst fur Gott und der Christenheit Jhrer handlung rechenschaft geben; aber unsern glauben, darbey wir bis her geblieben und hinfürder mit gottlicher hulf bis an unser nur gedenken zu bleiben, baben wir mit warhaftigem herzen in dieser schrieft bekant and dargetan.

Bitten hiemitt den lieben Gott. er wolle uns allezeigt durch seynen heiligen geist darbey erbalten und schutzen; auch begeren wir von allen fromen Christen, sie wollen in betrachtung des gottlichen willens und ernsten befelhs, der also lautet: Du solt nicht falsch gezengnis reden wider deynen nechsten, Keynen afterreden, so diesen unsern warhaftigen bericht und bekantnu $B$ zu wider und $z u$ unbilliger rorkleynerang unsers christlichen und Furstlichen namens hin und wider ausgebreitet werden, glauben noch stat geben, sondern viel mehr unser unschult wider dieselben nach dem wie billig, helfen retten, Daran geschieht Gotts des almechtigen wolgefelliger will, and wir seind dasselb gegen ein Jden, wes standes und wirden er sein mag, freundlich gnediglich und Christlich zu beschulden geneigt und erbotig.

Datam Königsberg den 13. Julii Anno 1554. 